\title{
Assessment of the Effectiveness of Coffee De-mucilager and Driers for Physical and Sensorial Coffee Quality
}

\author{
Mikru Tesfa ${ }^{1, *}$, Abrar Sualeh², Nigussie Mekonen ${ }^{2}$ \\ ${ }^{1}$ College of Agriculture, Food and Climate Science, Injibara University, Injibara, Ethiopia \\ ${ }^{2}$ Jimma Agricultural Research Centre (JARC), Jimma, Ethiopia \\ Email address: \\ mikrutesfa27@gmail.com (M. Tesfa), mabrarsualeh@yahoo.com (A. Sualeh), abalenm@gmail.com (N. Mekonnen) \\ ${ }^{*}$ Corresponding author
}

\section{To cite this article:}

Mikru Tesfa, Abrar Suleh, Nigussie Mekonen. Assessment of the Effectiveness of Coffee De-mucilager and Driers for Physical and Sensorial Coffee Quality. World Journal of Food Science and Technology. Vol. 5, No. 2, 2021, pp. 33-36. doi: 10.11648/j.wjfst.20210502.13

Received: April 6, 2021; Accepted: May 27, 2021; Published: June 16, 2021

\begin{abstract}
Coffee Arabica is an essential commodity to the livelihood of millions of Ethiopians and its quality has critical importance to the coffee industry. The objective of the present study was to evaluate the effect of newly introduced coffee postharvest machineries and driers on coffee raw, cup and total quality as compared to the conventional processing methods. Different methods, such as normal fermentation (conventional) and drying in sun, normal fermentation and drying in artificial drier, normal fermentation dried in poly tunnel drier, demucilager pulped and dried in sun, demucilager pulped and dried in artificial drier and demucilager pulped dried in poly tunnel were used for coffee sample preparation. The experiment was conducted during the 2017/18 and 2018/19 cropping season at Limmu Kossa coffee plantation. Both raw and cup quality parameters were evaluated by a team of certified panelists at Jimma Agricultural Research Center (JARC) coffee processing and quality analysis laboratory and analyzed using completely Randomized Design (CRD). Physical coffee quality parameters such as Bean size, Bean shape and make, color and odor showed no statistically significant $(\mathrm{P}>0.05)$ difference for all preparation methods. The highest values of cup quality parameters like Acidity (AC), astringency (AS), bitterness (BI), body (BO), flavor (FL) and overall quality (OAQ) were recorded for coffee samples prepared using demucilager pulper and dried in tunnel drier. Samples pulped by demucilager and dried in poly tunnel drier exhibited the highest values, 35.67, 47.50 and 83.17, for raw, cup and total quality, respectively. It seems important to further study the biochemical composion of samples prepared using in such new introduced demucilager and driers in the future.
\end{abstract}

Keywords: Artificial Drier, Demucilager, Poly Tunnel Drier

\section{Introduction}

Coffee is the major source of foreign currency for Ethiopia and contributes more than $35 \%$ of the total export earnings [6]. Thus, it is a cornerstone in the export economy of the country and it supports directly or indirectly the livelihood of some 15 million people [5]. Coffee processing is a very important activity in coffee production system and plays a crucial role in quality determination [7]. The quality of Ethiopian coffee is determined by two main factors, namely geographic origin and postharvest processing techniques [8].

Physical and organoleptic qualities are the most important parameters in the world coffee trade. It is estimated that $40 \%$ of the quality of coffee is determined in the field (Agricultural practices), $40 \%$ at postharvest primary processing, and $20 \%$ at export processing and handling, including storage [8]. This underscores the importance of primary processing in enhancing the quality and value of Coffee. For Ethiopian coffee, natural fermentation is recommended as it improves both raw and roast qualities [3]. However, natural fermentation is time consuming and costly, causes weight loss and is laborious compared to demucilager. Moreover, natural fermentation requires strict supervision on adjustment of temperature and time of fermentation, use of good quality equipment's and substantial amount of water (with acceptable quality) in order to get a quality coffee product. On the other hand, demucilager is found to be less costly, less time consuming and less laborious and does not require close supervision. Moreover, demucilager was reported to avoid 
coffee weight loss compared to natural fermentation. Very recently, different coffee processing stations are employing demucilager in the major coffee growing areas of Ethiopia taking into account its advantage in handling and cost effectiveness. According to some studies in Kenya, Costa Rica and Colombia, both natural fermentation and demucilager showed no significant influence on the final coffee quality [10] However, information on the effects of high-tech processing machineries (demucilager) on coffee quality of improved Ethiopian coffee varieties is scanty.

Coffee quality problems are mainly associated with improper post-harvest processing and handling practices such as drying on bare ground, improper wet processing, storage and transportation, poor agronomic practices like uncontrolled shade level, lack of stumping, pruning and weeding and poor harvesting practices, such as stripping and collecting dropped fruits from the ground [4].

Risk of contamination is greater during fermentation due to miss use of optimum fermentation time, lack of clean water, bean-soil contact during sun drying and bean rehumidification under high relative humidity conditions. These problems could be eliminated with demucilager pulper, optimum fermentation time and the use of unpolluted water and also artificial drying. However, artificial drying is an operation of high energy demand and it has been shown that if the bean's temperature exceeds $45^{\circ} \mathrm{C}$ during drying, the coffee's quality would be harmed [9]. Among the problems in post-harvest processing and handling in the study area, wet coffee washing after fermentation to facilitate the removal of mucilage prior to soaking pulped coffee for 24-48 hours to be fermented naturally in a concrete storage tanks is the major one that affects coffee quality. The fermentation is followed by washing through scrubbing the soaked beans against the concrete floor, which resembles 3 to 4 rinses of clean water to remove all traces and decomposed products of the mucilage. This process is time consuming and laborious. [1]. currently, different new machines remove the skin and pulp from coffee cherries leaving viscous mucilage adhering to parchment. However, there is limited information on the effect of the new technologies on physical and sensory characteristics of coffee brew. Therefore, the objective was to investigate the effect of newly introduced coffee postharvest machineries and driers on coffee raw, cup and total quality as compared to the conventional processing methods.

\section{Materials and Methods}

\subsection{Study Area}

The experiment was conducted in Limmu Kossa sites of the Limmu Coffee Plantation Development Enterprise. Limmu Kossa woreda is located at $7^{\circ} 50 \mathrm{~N}$ latitude and $36^{\circ}$ 53 E longitudes in Southwest part of Ethiopia and $421 \mathrm{Km}$ far from Addis Ababa. It has an area of $2770.5 \mathrm{Km}^{2}$. The area has annual rainfall varies between 1200 and $1850 \mathrm{~mm}$ with mean minimum and maximum temperatures of $7^{\circ} \mathrm{C}-12^{\circ} \mathrm{C}$ and $25^{\circ} \mathrm{C}-30^{\circ} \mathrm{C}$, respectively.

\subsection{Experimental Materials}

Six coffee bulk prepared samples by different preparation methods.

\subsection{Experimental Procedures and Design}

For the purpose of uniform processing, only well ripe red coffee cherries were selectively harvested at peak harvesting time. Six different samples were prepared such as normal fermentation (conventional) dried by sun, normal fermentation dried by artificial drier, normal fermentation dried by poly tunnel drier, demucilager pulped dried by sun, demucilager pulped dried by Artificial drier and demucilager pulped dried by poly tunnel were performed and analyzed by CRD (Completely Randomized Design).

\subsection{Sample Preparation}

At Limmu Kossa coffee plantation farm six coffee samples of $8 \mathrm{~kg}$ per sample were prepared based on our treatment and used.

\section{Data Collection}

\subsection{Raw Quality Evaluation Data}

During raw quality analysis, $100 \mathrm{~g}$, of green bean was used for each sample and their screen bean size, shape and make, color and odor were evaluated by a team of JARC cuppers according to JARC coffee quality liquoring laboratory manual [2].

\subsection{Roasting and Grinding}

Hundred gram of green coffee bean per sample was used for roasting. Each sample was separately roasted until the bean attain medium brown roast color for (8 minutes on average) by using roaster machine (Probat BRZ6, Germany) with a cylinder heat of $180-200^{\circ} \mathrm{C}$. When roasting was considered medium, the coffee beans were tipped out in to the cooling tray and allowed to cool for about 4 minutes. After cooling and before grinding, the samples were blown to remove the loose silver skins (chaff).

Half of roasted bean from total roasted sample per each sample was grounded immediately before brewing using electrical coffee grinder (MahlKonig, Germany) with middle adjustment to get medium size coffee powder.

\subsection{Brew Preparation}

Three cups of same volume $(180 \mathrm{ml})$ and size were used for each sample to assess cup quality medium level grinded coffee powders weighing $8 \mathrm{~g}$ were placed in each cup for liquoring.

Boiled water was poured into half volume of the cup containing $8 \mathrm{~g}$ of coffee powder, and thereafter the volatile aromatic quality and intensity parameters were recorded by sniffing by well trained and licensed coffee quality testers. 
Then, the cups were filled with boiled water to the brim and left to cool for 3 minutes. Then after, the foam of the beverage of each cup is skimmed off with spoon. Finally, the brew was made ready for panelists within 8 minutes.

\subsection{Cup (Organoleptic) Quality Evaluation Data}

This was also carried out at JARC coffee liquoring laboratory using the standard procedures. Once the beverage was cooled to around $60^{\circ} \mathrm{C}$ (palatable temperature), then cup tasting was carried out by the three trained panelists and licensed Q graders. Three cups per sample were prepared for a tasting session arranged randomly. Samples were chosen randomly for testing.

Testers noted sensorial quality attributes following the format and procedure developed at Jimma Agricultural Research Center [2]. Aroma (aromatic quality and intensity), Astringency and Bitterness were scored using scales ranging from 0 to 5 . Body, acidity, flavor and overall quality were scored using scales ranging from 0 to 10 . Typical flavor (Typicity) was assessed as an after taste aromatic quality that could vary from winy to flowery (winy, fragrant, floral, citrus, mocha, fruity and spicy). Mean of each variable by the panel was used for statistical analysis.

\section{Statistical Analysis}

Analysis of variance (ANOVA) was performed to determine the effects of recently introduced coffee postharvest machineries on coffee qualities. Least Significant Difference (LSD) was used for the mean separation.

\section{Results and Discussions}

\subsection{Physical (Raw) Coffee Quality Attributes}

Physical coffee quality attributes (bean size, bean shape and make, color and odor) are presented in Table 1. There was no significance difference $(P>0.05)$ among the preparation methods for bean size, bean shape and make, color and odor. The raw total quality also showed no significant $(\mathrm{P}>0.05)$ among samples prepared in different preparation methods. The lowest value of odor (8.67) was recorded for samples prepared by normal (conventional) fermentation and dried using artificially drier.

Table 1. Effect of recently introduced post-harvest machineries and drier on physical (raw) quality attributes of coffee.

\begin{tabular}{|c|c|c|c|c|c|}
\hline \multirow{2}{*}{ Preparation method } & \multicolumn{5}{|c|}{ Green bean coffee quality parameters } \\
\hline & Bean size screen & Shape and make & Color & Odor & RT $(40 \%)$ \\
\hline NFDS & $95.5 \mathrm{a}$ & $12.17 \mathrm{a}$ & $12.00 \mathrm{a}$ & $9.67 \mathrm{a}$ & $33.83 \mathrm{a}$ \\
\hline NFDA & $96.50 \mathrm{a}$ & $12.67 \mathrm{a}$ & $12.17 \mathrm{a}$ & $8.67 \mathrm{~b}$ & $33.50 \mathrm{a}$ \\
\hline NFTd & $97.20 \mathrm{a}$ & $12.00 \mathrm{a}$ & $12.33 \mathrm{a}$ & $10.00 \mathrm{a}$ & $34.33 \mathrm{a}$ \\
\hline DPDS & $95.50 \mathrm{a}$ & $12.83 \mathrm{a}$ & $13.00 \mathrm{a}$ & $9.67 \mathrm{a}$ & $35.50 \mathrm{a}$ \\
\hline DPDA & $98.33 \mathrm{a}$ & $12.83 \mathrm{a}$ & $12.83 \mathrm{a}$ & $10.00 \mathrm{a}$ & $35.67 \mathrm{a}$ \\
\hline DPTd & $95.00 \mathrm{a}$ & $12.67 \mathrm{a}$ & $12.33 \mathrm{a}$ & $10.00 \mathrm{a}$ & $35.00 \mathrm{a}$ \\
\hline $\operatorname{LSD}(0.05)$ & 1.60 & 0.54 & 0.60 & 0.33 & 1.05 \\
\hline
\end{tabular}

Mean values followed by the same letter with in a column are not significantly different $(\mathrm{P}>0.05)$.

NFDS, NFDA and NFTd=Normal (conventional) fermentation dried in sun, dried artificially and dried using tunnel drier respectively, DPDS, DPDA and DPTd=Demucilager pulped and dried in sun, dried artificially and dried using tunnel drier, respectively. RT=Raw total

\subsection{Organoleptic (Cup) Quality Attributes}

Results on the effect of recently introduced coffee postharvest machineries and drier on Organoleptic (cup) quality attributes are presented in Table 2. The highest values of cup quality parameters, like acidity (AC), astringency (AS), bitterness (BI), body (BO), flavor (FL) and overall quality (OAQ) were recorded for coffee samples prepared using demucilager pulper and dried in poly tunnel drier (Table 2). The highest (47.5) and lowest (43.0) values of total cup quality were recorded for samples prepared using demucilager pulper and dried in tunnel drier and conventional fermentation and dried in open sun, respectively. All prepared samples showed no statistically significant $(\mathrm{P}>$ 0.05 ) effect in body.

Table 2. Effect of recently introduced post- harvest machineries and driers on Organoleptic (cup) quality attributes of coffee.

\begin{tabular}{|c|c|c|c|c|c|c|c|c|c|}
\hline \multirow{2}{*}{ Preparation method } & \multicolumn{9}{|c|}{ Cup quality parameters } \\
\hline & AI & AQ & $\mathrm{AC}$ & AS & BI & BO & FL & $\mathbf{O L}$ & CT $(60 \%)$ \\
\hline NFDS & $3.67 b$ & $3.67 \mathrm{bc}$ & $7.17 \mathrm{~b}$ & $3.67 \mathrm{~b}$ & $3.33 \mathrm{c}$ & $7.33 \mathrm{a}$ & $7.00 \mathrm{~b}$ & $7.17 b$ & $43.00 \mathrm{c}$ \\
\hline NFDA & $3.67 \mathrm{~b}$ & $3.50 \mathrm{c}$ & 7.33ab & $3.67 \mathrm{~b}$ & $3.33 \mathrm{c}$ & $7.50 \mathrm{a}$ & 7.33ab & 7.33ab & $43.67 \mathrm{c}$ \\
\hline NFTd & $4.17 \mathrm{a}$ & $4.17 \mathrm{a}$ & $7.17 \mathrm{~b}$ & $3.83 \mathrm{ab}$ & $3.50 \mathrm{bc}$ & $7.17 \mathrm{a}$ & $7.00 \mathrm{~b}$ & $7.17 \mathrm{~b}$ & $44.17 \mathrm{bc}$ \\
\hline DPDS & $4.00 \mathrm{ab}$ & $4.00 \mathrm{ab}$ & $7.83 \mathrm{a}$ & $4.00 \mathrm{ab}$ & $4.00 \mathrm{ab}$ & $7.83 \mathrm{a}$ & $7.67 \mathrm{a}$ & 7.67ab & $47.00 \mathrm{ab}$ \\
\hline DPTD & $3.83 \mathrm{ab}$ & $3.83 \mathrm{abc}$ & $7.83 \mathrm{a}$ & $4.17 \mathrm{a}$ & $4.17 \mathrm{a}$ & $8.00 \mathrm{a}$ & $7.83 \mathrm{a}$ & $7.83 \mathrm{a}$ & $47.50 \mathrm{a}$ \\
\hline DPDA & $3.83 \mathrm{ab}$ & $3.67 \mathrm{bc}$ & 7.33ab & $3.83 \mathrm{ab}$ & $3.67 \mathrm{abc}$ & $7.33 \mathrm{a}$ & $7.50 \mathrm{ab}$ & 7.33ab & $44.50 \mathrm{bc}$ \\
\hline $\operatorname{LSD}(0.05)$ & 0.22 & 0.19 & 0.24 & 0.22 & 0.3 & 0.38 & 0.24 & 0.28 & 1.32 \\
\hline
\end{tabular}

Mean values followed by the same letter with in column are not significant difference $(\mathrm{P}>0.05)$.

$\mathrm{AQ}=$ aromatic quality, $\mathrm{AC}=$ acidity, $\mathrm{AS}=$ astringency, $\mathrm{BI}=$ bitterness $\mathrm{FL}=$ flavour, $\mathrm{OL}=$ overall quality and $\mathrm{CT}=$ cup total 
Table 3. Effect of recently introduced post-harvest machineries and driers on total quality value of coffee.

\begin{tabular}{llll}
\hline \multirow{2}{*}{ Preparation methods } & Total quality Values & & Overall quality value (100\%) \\
\cline { 2 - 4 } & Raw total (40\%) & Cup total (60\%) & $76.83 \mathrm{c}$ \\
\hline NFDS & $33.83 \mathrm{a}$ & $43.00 \mathrm{c}$ & $77.17 \mathrm{c}$ \\
NFDA & $33.50 \mathrm{a}$ & $43.67 \mathrm{c}$ & $78.50 \mathrm{bc}$ \\
NFTd & $34.33 \mathrm{a}$ & $44.17 \mathrm{bc}$ & $82.50 \mathrm{ab}$ \\
DPDS & $35.50 \mathrm{a}$ & $47.00 \mathrm{ab}$ & $83.17 \mathrm{a}$ \\
DPTd & $35.67 \mathrm{a}$ & $47.50 \mathrm{a}$ & $79.50 \mathrm{abc}$ \\
DPDA & $35.00 \mathrm{a}$ & $44.50 \mathrm{bc}$ & 2.83 \\
CV $(\%)$ & 3.73 & 2.83 & 1.84 \\
LSD $(0.05)$ & 1.05 & 1.32 & \\
\hline
\end{tabular}

Mean values followed by the same letter with in a column are not significant difference $(\mathrm{P}>0.05)$.

\subsection{Total Quality Values}

Though no statistically significant difference $(\mathrm{P}>0.05)$ was observed among samples processed and dried in different preparation methods on raw quality values shown as (Table 3). Samples pulped in demucilager and dried in poly tunnel drier was recorded the highest values 35.67, 47.50 and 83.17 for raw, cup and total quality, respectively.

\section{Conclusion}

There was no statistically significant difference $(\mathrm{P}>0.05)$ among coffee samples processed and dried in different preparation methods for raw quality. Samples pulped by demucilager and dried in poly tunnel drier exhibited the highest values, $35.67,47.50$ and 83.17 , for raw, cup and total quality, respectively. Generally the result indicated that out of the six coffee samples assessed for raw, cup and overall quality, those demucilager pulped and dried in open sun (DPDS) and demucilager pulped and dried in poly tunnel drier (DPTd) showed very good overall quality standard and were found to be highly acceptable. So using newly introduced demucilager and drying technologies had no negative impact on both raw and sensory quality attributes and fit to export standards of samples and were found to be less time consuming and less laborious. However, further study like evaluating of biochemical component, cost benefit analysis should be carried out to generate complete information on the processing methods and similar experiments should be carried out in some major coffee growing areas of the country to come up a comprehensive conclusion.

\section{Acknowledgements}

We acknowledged the Ethiopian Institute of Agricultural Research (EIAR) and Horizon coffee plantation PLC for financial supports and sample preparation, respectively during the study period. We are also thankful to the team of JARC coffee cuppers.

\section{References}

[1] Anwar Abasanbi. 2010. Assessment of coffee quality and its related problems in Jimma zone of Oromia regional state. M. Sc. Thesis, Jimma University, Ethiopia.

[2] Abrar Sualeh and Negussie Mekonnen. 2015. Manual for Coffee Quality Laboratory ISBN: 978999446605410-8.

[3] Behailu Wolde Senbet, Abrar Sualeh, Nigusie Mekonen and Solomon Endris. 2008. A review of coffee Processing and Quality Research in Ethiopia. In: Coffee Diversity and Knowledge. Ethiopian Institute of Agricultural Research, pp: 307-316.

[4] Dessie Nure. 2008. Mapping quality profile of Ethiopian coffee by origin. In: Proceedings of a National Work Shop four decades of coffee research and development in Ethiopia. 14-17 August 2007, EIAR, Addis Ababa, Ethiopia. pp. 328333.

[5] EEA (European Environment Agency). 2001. Annual report on Ethiopian economy. 1999/2000, EEA, Addis Ababa, Ethiopia.

[6] FAO/WFP. 2008. Special report FAO/WFP crop and food supply assessment mission to Ethiopia. 24 January 2008.

[7] Mburu, J. K. 1999. Notes on coffee processing procedures and their influence on quality. Kenya coffee, 64 (750): 2861-2867.

[8] Musebe, R. C. Agwenanda and M. Mekonnen, 2007. Primary Coffee Processing in Ethiopia: In Africa Crop Science Society; Africa Crop Science Conference Proceedings, 8: 1417-1421.

[9] Sfredo M. A. Finzer J. R. D. and Limaverde J. R. (2005). Heat and mass transfer in coffee fruits drying. Journal of Food Engineering 70, 15-25.

[10] Wintegens, J. N. 2004. Coffee: Growing, processing, sustainable production, a guide book for growers processors, traders, and researchers, WILEY - VCHVerlag GmbH \& Co. $\mathrm{KGaA}$, Weinheium. 